\title{
Fourth-order improved Runge-Kutta method for directly solving special third-order ordinary differential equations
}

\begin{abstract}
In this paper, fourth-order improved Rungeï Kutta method (IRKD) for directly solving a special third-order ordinary differential equation is constructed. The fourth-order IRKD method has a lower number of function evaluations compared with the fourth-order Rungeï Kutta method. The stability polynomial of the method is given. Numerical comparisons are also performed using the existing Rungeï Kutta method after reducing the problems into a system of first-order equations and solving them, and direct RKD method for solving special third-order ordinary differential equations. Numerical examples are presented to illustrate the efficiency and the accuracy of the new method in terms of number of function evaluations as well as max absolute error.
\end{abstract}

Keyword: Special third-order; Ordinary differential equations; Rungeï Kutta method; IRKD method 